\title{
Nurturing Natural Sensors
}

\author{
Stacey Kuznetsov, William Odom, James Pierce, Eric Paulos \\ Human-Computer Interaction Institute \\ Carnegie Mellon University \\ Pittsburgh, PA, USA \\ \{stace, wodom, jjpierce, paulos\}@cs.cmu.edu
}

\begin{abstract}
Sensing has played a significant role in the evolution of ubiquitous computing systems, enabling many of today's compelling interactive and ubiquitous experiences. In this paper, we argue for expanding the current landscape of sensing to include living organisms such as plants and animals, along with traditional tools and digital devices. We present a field study of ten individuals who routinely work with living organisms such as plants, fish, reptiles and bees, and rely on these organisms as well as analog instruments and digital sensors to infer environmental conditions and inform future actions. Our findings offer a new perspective on everyday biomarkers, and we use the lens of organic and non-digital sensing to reflect on current sensing paradigms in ubiquitous computing. We conclude with three opportunity areas to help frame future work in ubiquitous sensing: (1) incorporating traditional technologies and living systems into ubiquitous sensing applications, (2) developing information technologies that teach new ways of 'seeing', and (3) supporting richer forms of metadata to unite stakeholders through their actions, interests and concerns.
\end{abstract}

\section{Author Keywords \\ Phenology, biomarkers, sensors}

\section{INTRODUCTION}

Over the past few decades, UbiComp and HCI communities have explored a range of sensing systems to support our interactions with local environments, as well as the people, technologies and artifacts inhabiting them. While a sensor can be broadly defined as any device that responds to a physical stimulus, the majority of prior and ongoing research in UbiComp has understandably focused on electronic instantiations of sensing devices. In this paper, we present the practices of gardeners, beekeepers, zoologists and other 'experts' in the domain of organic and non-digital sensing to reflect on the question, when is an electronic sensor appropriate or necessary in a given context?

Visionary research has often turned to groups outside 'mainstream' user populations to productively inform new areas of inquiry within the UbiComp community [e.g., 39]. Similarly, we explore the values and practices of

Permission to make digital or hard copies of all or part of this work for personal or classroom use is granted without fee provided that copies are not made or distributed for profit or commercial advantage and that copies bear this notice and the full citation on the first page. To copy otherwise, or republish, to post on servers or to redistribute to lists, requires prior specific permission and/or a fee.

UbiComp '11, Sep 17-Sep 21, 2011, Beijing, China.

Copyright 2011 ACM 978-1-60558-843-8/10/09...\$10.00. individuals who use everyday biomarkers- common biological organisms that express information about an ecosystem or its many parts. We present a field study of 10 participants who routinely work with living organisms such as plants, fish, reptiles or bees. While many people make inferences about the environment (e.g., a cloudy sky suggests the possibility of rain), we expect our sample of participants to be more attuned to environmental processes as their work explicitly engages with living systems. Specifically, we focus on participants' use of digital devices, traditional tools and living organisms to infer environmental conditions and inform actions related to local ecosystems. In doing so, we reflect on current sensing paradigms in ubiquitous computing through the lens of organic and non-electronic sensing.

Our findings offer new insights into everyday biomarkers and serve to expand UbiComp visions of sensing to include more traditional instruments as well as the living organisms themselves. We conclude with three opportunity areas to help critically frame future work in ubiquitous sensing: (1) leveraging non-digital sensors, (2) designing technologies that teach new ways of 'seeing', and (3) enriching practices of data collection and sharing.

\section{WHAT IS A SENSOR?}

In what follows, we present several categories of electronic sensing technologies that emerged from our review of the UbiComp and HCI literature. Although these categories are by no means exhaustive or exclusive, they help contextualize the diverse range of sensors currently studied by these communities.
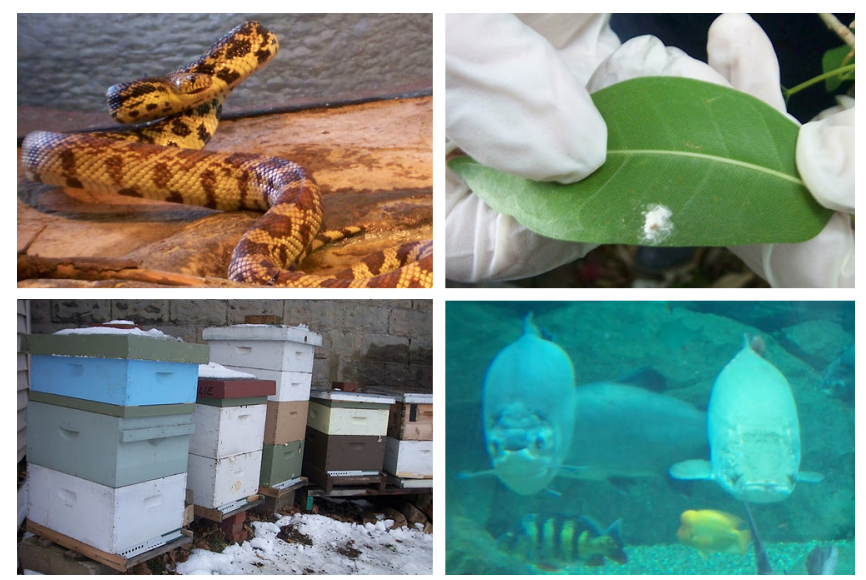

Figure 1. Everyday biomarkers: reptile posture suggesting a disturbance to the environment (top left); scale larvae signifying a pest problem (top right); bee behavior reflecting local weather and bloom cycles (bottom left); fish appearance indicating water quality and parasite levels (bottom right). 


\section{Input techniques}

Early visions of sensing in HCI focused on detecting human actions to manipulate computer systems. For instance, Card, et al.'s taxonomy of input devices [8] quantifies sensing in terms of expressiveness and effectiveness. Kurtenbach and Buxton emphasize 'direct manipulation', contributing a suite of touch and gesture sensing technologies [24]. More recently, novel input techniques have appropriated everyday objects, surfaces or the body to capture human input [e.g., 19]. Many of these new, more natural input techniques fall under the moniker of Natural User Interfaces (NUI). To be clear, "natural" terminology used in NUI refers to the interactive experience "feeling natural" (not to natural organisms as parts of the system).

\section{Activity recognition/ infrastructure sensing}

Another area of research explores sensing human behavior implicitly. For instance, wearable sensors support tracking routine actions [27]; or sensor-enabled mobile phones monitor physical exercise [10]. Ubiquitous systems are also designed to infer human behavior by sensing surrounding infrastructure, for example by sensing and tracking interactions with common appliances in the home $[16,18]$.

\section{Active participatory sensing}

New, often low-cost and DIY (do it yourself) technologies enable people (typically 'non-experts') to actively measure a range of environmental factors through participatory sensing [40]. Examples include handheld air quality monitors $[14,30]$, or mobile phones for tracking water quality [22], among many other others.

\section{Passive environmental monitoring}

An alternative body of sensing applications does not require continuous human involvement to monitor the environment. Examples include air quality monitors deployed on street sweepers [1] and autonomous robots [21]; or sensors left in public spaces [26] and private homes [23]. Here, humans may still interact with the collected data (e.g., via map or graph) without explicitly engaging in the sensing process.

\section{RELATED LITERATURE}

As interest within the sensing community expands beyond indoor settings (home or office), new technologies are designed to promote deeper engagement with urban and rural environments. New applications are emerging to augment human interactions with organic materials, including sensor networks to support agricultural production in vineyards $[3,6]$, networks for non-invasive quality inspection of agricultural produce [37], or smart garden watering to promote conservation practices [30]. Other research focuses on context-aware computing to offer agricultural information [1], and, more generally, the educational potential of mobile sensor-based applications for learning about outdoor environments [7, 35, 38].

Critiques of sensing technologies emphasize expanding the scope of environmental sensing and, in some cases, drawing attention to possible unintended consequences. Aoki et al. [1] illustrate how the framing of environmental sensing and data collection can be expanded to encourage participation in public discussion of political issues. Similarly, DiSalvo et al.'s [14] review of related works articulates how political participation represents a key future dimension for sensing-based research to consider. Leshed et al. [28] critically investigate how GPS navigation systems can disengage people from the environments through which they navigate. Others describe the potential unproductive outcomes technical interventions might have on community organization and practices that otherwise sustain smallscale food production efforts [e.g., 29].

\section{Research exploring existing practices}

Another body of research explores existing practices in order to inform the design of future ubiquitous technologies [e.g., 41]. For example, Chetty et al. [9] and Dillahunt et al. [11] both study home resource consumption to draw out implications for the design of ubiquitous energy sensing; and Wyche, et al. examine how Pentecostals use communication technologies, suggesting interventions for supporting alternative value systems in UbiComp [42]. We build on these respective works by exploring the tools and practices of individuals working with living systems.

\section{METHODS}

We conducted semi-structured interviews (2-3 hours) with 10 participants who work with organic organisms (plants, reptiles, bees, fish, etc.) in and around a mid-sized city in the United States. Participants (ages 20's to late 60's; 3 female, 7 male) were recruited through local gardening and beekeeping communities, and the city zoo. Interviews took place at participants' respective sites of work (garden, zoo, apiary, etc.) to support rich, in situ reflections. We asked participants to walk through their daily routines (with regards to gardening, beekeeping, etc.) and, in doing so, show us their tools and local settings. Additionally, openended questions probed common uses of digital and nondigital sensors (and other technologies, such as computers, mobile phones, etc.); participants' knowledge of the environment based on tools and living systems; and how this information is shared with fellow practitioners or other stakeholders.

We audio recorded all interviews and took field notes, documentary photographs and select videos. The research team repeatedly reviewed the audio and all field materials to draw out underlying themes. Interview audio recordings were transcribed and coded using these themes. We also created conceptual models and affinity diagrams to reveal themes and unexpected connections across our data.

\section{FINDINGS}

We begin our findings by introducing the participants. We then detail our data in regards to: 1) monitoring practicesparticipants' use of technology, tools and observation to monitor the environment; 2) types of living indicatorshow participants use living organisms as environmental indicators; 3) collection, sharing and speculation - patterns of discussion and speculation around biomarker data.

\section{Participants}

We recruited 10 diverse participants who routinely work with living organisms, including: beekeepers who maintain hives at home and throughout the city as a part of local 
community initiatives; an Integrated Pest Management (IPM) scientist who controls pest populations in a tropical greenhouse through organic means, such as beneficial insects that consume pests; a horticulturist who innovates, builds and maintains bioshelters (self-sustaining greenhouses, "designed on the model of a cell- like a living cell that looks like a living organism", H); organic farmers and gardeners who work independently or with urban agriculture groups; and reptile and fish overseers at a local zoo that acutely examine animal behaviors to inform their daily work. We reference data owing to individuals from each domain as follows:

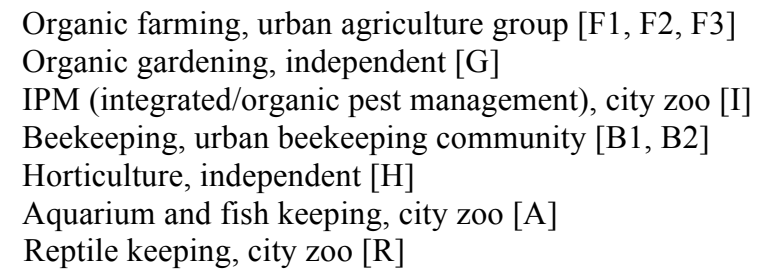

\section{Participants' backgrounds}

All participants have worked in their domain for several years, and most have been involved for decades: 8 participants have lived on farms or worked in gardens, zoos or aquariums for over 20 years. 7 participants earned professional degrees in biology, sustainable community development, etymology, zoology or a related field. However, all participants emphasize learning their craft through a mentor ("Beekeeping is kind of an art or a craft, you really do need a mentor... you really need somebody local that can really tune you in with what's going on locally", B1) or hands-on experience ("just a function of working with fish a really long long time-you start to get kind of a zen feel for, ok something is not right there", A).

\section{Motivations}

Participants rely on their work for income, either by selling equipment or produce, through education initiatives (radio shows, books, etc.), or employment at an urban agriculture community or the city zoo. However, they also emphasize enjoyment ("I enjoy growing-personally that's the most rewarding thing for me", F1; "the fact of seeing something go from just a little ity bity thing all the way to fruition", G) and the broader benefits of their practice ("every third bite of food that we eat as a society is attributed to the honey bee", B1) as primary motivations behind their work.

\section{Monitoring practices}

Despite differences in their specific domains, all participants' work revolves around regular check-ups to track the health of the organisms their work relates to. From daily 'rounds' at tanks and aquariums ("The first thing we do when we start is to check on our animals, we do rounds", R; "You just stand and watch them [fish] for a little bit and you see... Am I noticing anything that's off", A) to weekly inspections of select plants ("Every Wednesday we go and inspect certain plants", I) or the bi-weekly examination of beehives ("I check my hives once in 12 to 14 days", B2), participants routinely monitor their environment. We continue by detailing participants' monitoring practices, organized by technological complexity, starting with practices that rely on technology as a primary means of data collection, and ending with practices that draw on more traditional instruments or do not involve any tools ('naked' human observation).

\section{Technology-mediated monitoring}

Predictably, some monitoring practices fundamentally rely on technology (Figure 2). Digital sensors are used routinely, occasionally (to clarify an anomaly), or early on (before participants acquire a skill and abandon the technology).

\section{Routine digital sensing}

Participants draw on certain technologies on a regular basis. A thermometer is used twice a day in aquariums ("if you can catch the temperature before the chiller has been off for too long maybe you can save the animals", A); daily at greenhouses, reptile tanks and bioshelter ("if it's cold [we] build the fire and open and close vents as needed", H), and weekly in IPM:

We try to look at the temperature and humidity and see how that affects the population of pests... we know that certain insects like spider mites, they love hot dry temperature. (I)

Weather is also checked regularly (online, TV, etc.), for instance, to infer soil conditions ("we're always looking at the weather, like you shouldn't do this when the ground is wet, you should do this", F2) or availability of pollen:

I watch to see what the weather is like: the flowers may be open, but maybe raining and the plant flowers can't secrete nectar, the bees can't get to the flowers, maybe too cold maybe very dry, there's not an excess moisture for the flowers to secret a lot of nectar. (B1)

Moreover, A checks ORP sensors twice a day to ensure proper function of the ozone generator ("check in's and check out's. Spend a good hour and a half of my day", A) and runs water quality tests weekly ("we do monitor it [water] once a week to make sure the carbon is still removing the chlorine", $\mathrm{A})$. $\mathrm{R}$ uses a scale to weigh reptiles while they are quarantined ("we do weights, measurements,
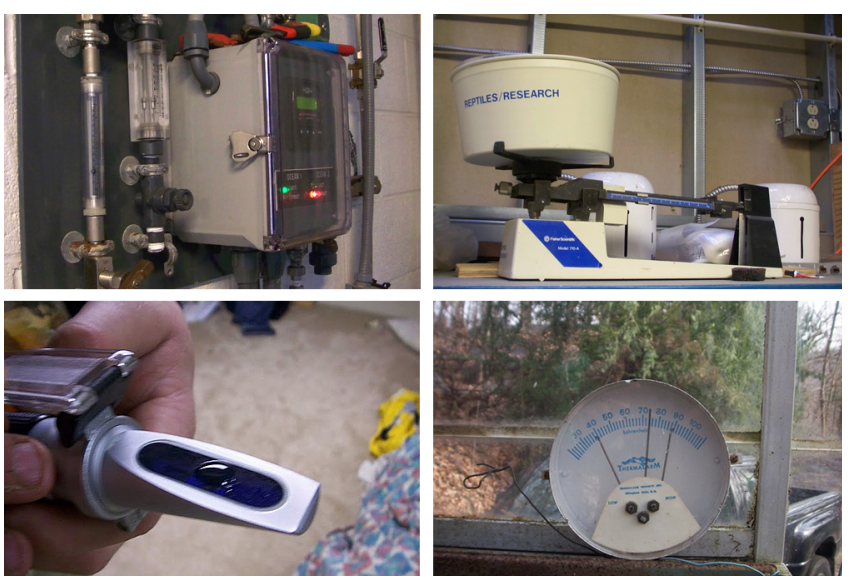

Figure 2. Technologies used by participants: ORP probe, routinely used to monitor ozone levels in aquariums (top left), scale for weighing reptiles (top right), refractometer, occasionally used to check honey before harvesting (bottom left); thermometer, checked daily (bottom right). 
we want to make sure the animal is thriving in quarantine", F). F1, F2 and F3 take annual soil tests as part of their farming community's standards but do not notice drastic differences as most soil is imported compost ("the nutrients would change a little bit, your nitrogen would jump up and down cause plans use to much of it", F1).

\section{Occasional digital sensing}

In addition to the digital tools employed during routine monitoring, some technologies are used only occasionally, to clarify an anomaly or confirm an observation. For example, although $\mathrm{G}$ does not routinely test the soil, he would use a test if his plants were not growing properly:

I've never tested the soil here, I'm getting everything I want out of my garden so I'm not gonna worry about it. It's more if you're having problems... if your beets aren't heading up, you know probably a $\mathrm{pH}$ problem or a nutrient deficiency [test the soil to] figure out what it is. (G)

Similarly, when F1 noticed dust accumulating from nearby dump trucks, he sent a sample to a local lab for testing:

The biggest air quality concern is the dump trucks full of slag that drive by... there's this like black dust that collects in the street and we scooped some of that up recently and sent it away for testing. I'm curious to see... (F1)

The aquarist participant relies on water quality testing when he notices unusual fish behavior ("we just start checking everything to see where's the problem, what's wrong", A). Water quality sensors include a $\mathrm{pH}$ meter, a spectrophotometer, which can run up to "400 different chemical analysis", and a dissolved oxygen meter:

We have a dissolved oxygen meter in the lab, we don't go around and check all the exhibits once a week... if there's a problem... we'll bring the DO [dissolved oxygen] meter out. We'll check that it's not too low or too high. (A)

Likewise, before harvesting honey, B2 occasionally uses a handheld refractometer to check its water content ("anything above 18\% tends to ferment, anything below $18 \%$ doesn't ferment", B2). Thus, while technologies such as a DO meter or refractometer are not part of routine practice, participants tend to draw on them when their observations suggest ambiguous outcomes.

\section{Abandoned digital sensing}

Lastly, in several instances, digital sensing is used early on but is eventually abandoned. F2 no longer uses a sprinkler timer since it caused a pipe to "burst", and participant I does not trust the Fogstat system to correctly water the greenhouse due to faulty humidity sensing ("it hasn't been calibrated in a while so it's way off", I). Additionally, some sensors were abandoned when a participant acquired a certain skill. For instance, participant I stopped testing her soil when she learned to infer soil quality from plant growth:

I used to [test soil] every year but it kept coming back pretty much the same. I guess I'm just sort of working off how everything looks- everything seems to be green and growing pretty well. (I)

Our findings thus suggest that while some technologies are regularly or occasionally used to monitor the environment, several types of sensors have been abandoned either due to malfunction or lack of useful data once a skill was acquired.

\section{Traditional tools and observation-based monitoring}

We discovered that a wide range of participants' monitoring practices do not involve digital sensing to understand environmental processes and states. Participants rely equally (although in different ways) on naturally occurring phenomena and non-digital 'measurement' tools.

\section{Magnification and counting tools}

Participants routinely use counting and magnification tools to infer environmental conditions (Figure 3). In beekeeping, a "monitoring tray" - a tray imprinted with a square inch grid - is placed under a hive. B2 accesses the infestation by counting the number of mites (pests) fallen on each square:

This is a monitoring tray... I'll slide it in there [under a hive]... We can either do a 24-hour count or a $15 \mathrm{~min}$ count. I just do a 15 min count. I'd look at it, and go ok there's 6 [mites] in that one and on average there's 5 or 6 mites, which is heavy per square... So then I can determine whether I want to do a treatment. (B2)

Similarly, F2 uses handmade traps (notecards covered with a sticky substance) to monitor pests based on the amount of caught insects:

"To see them [pest insects], we set sticky traps... you can go and you stick them in the dirt and you can go and see what you have... I don't count them [insects], but I kinda look at it. Like what's been caught on these cards-[F2 looks at card] it's not a ton, but it's definitely a lot." (F2)

In IPM, a magnifying hand lens helps observe larvae stages on leaves to determine if beneficial insects are thriving:
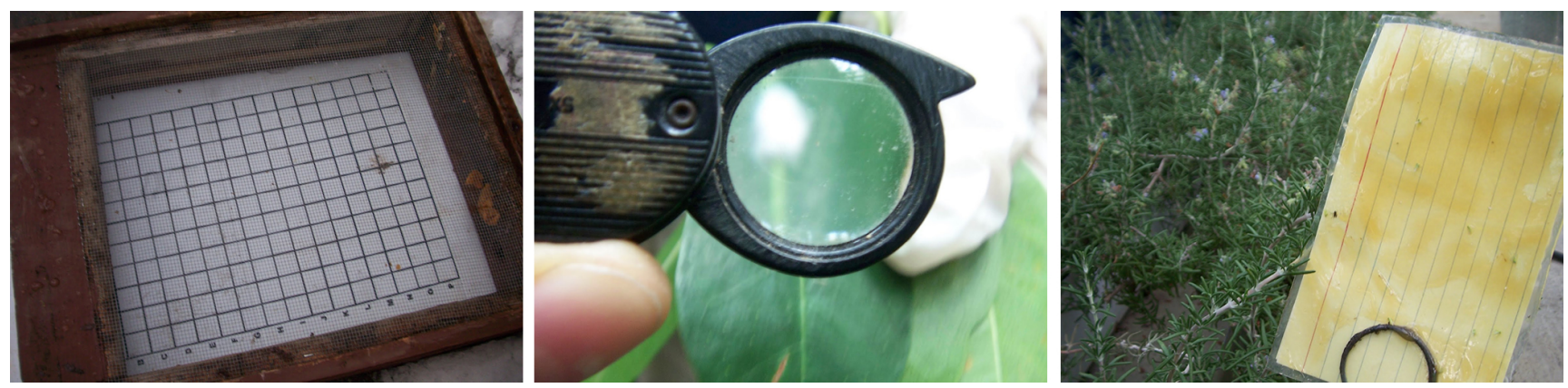

Figure 3. Magnification and counting tools: monitoring tray (left); hand lens (center); handmade sticky trap (right). 
If you look at this [larvae] under a hand lens, it has a really white wooly covering to it... getting to know the larval stages helps recognize that your beneficial insect is getting established. (I)

Likewise, high-resolution magnification is used to monitor fish and reptiles. A microscope is used for annual checkups ("the vet department will look at it [fecal matter] under a microscope and look for various parasites", R), or inspections of fish, often cross-referenced with "pictures of different parasites":

We'll open up the gill cover take a little snip of the filament put that on the slide with the skin scrape look at it on the scope look for things that are crawling around. (A)

Participants thus regularly use magnification and counting tools to reveal information that is invisible to the naked eye.

Monitoring through physical engagement and action In another set of practices aimed at exploring information that is not accessible through passive observation (hearing, sight, etc.), participants become active agents in their environment. For instance, the whitefly pest tends to reside on leaves and is not easily discernable to the naked eye. To access this infestation F2 habitually taps her plants, causing noticeable clouds of whitefly to emerge ("they [whitefly] come off in like a big cloud when you run your hand over it [plant]", F2). Similarly, since nectar is not visible from outside the hive, B1 and B2 routinely 'tip' each beehive to gauge its weight and infer whether the amount of nectar is sufficient:

You just pick up-you tip the hive. You pick up one end and you can tell by the weight how many stored pounds of honey there is in the hive and that's something we do this time of the year to see if the bees are starving. (B1)

In the bioshelter, $\mathrm{H}$ uses his finger to track moisture at various soil depths, accessing 'data' that might be unreliable or inaccessible through other means:

The human skin is a lot more sensitive than the gauges [moisture sensors]. When you want to know how moist the soil is at the bottom of the pot or the top of the pot... stick your finger in the pot and you know how far down it is. $(\mathrm{H})$

Our aquarist participant shares an analogous practice, scuba diving into exhibits to monitor aspects of fish appearance and behavior that might be invisible from outside the tank:

I would love to be in my exhibits more... when I go scuba diving I can get this close to the animal, I can see a lot more. Because of the way the windows filter the light and the animals react to the light and everything... there are things you just miss that if you get in you can see. (A)

These and other examples across all of our studied practices suggest physical action (tapping, tipping, inserting, etc.) as a means of gathering richer information, inaccessible through 'naked' and passive observation.

\section{Monitoring through 'naked' observation}

Finally, participants regularly infer information about the environment through 'naked' observations. Our findings include examples of the use of all five senses: smell ("if the water is really bad you can actually smell the ammonia", A); sight ("parsnip starts to sprout so that usually means the soil gets to 70 degrees... by [seeing] what's germinating I can tell the temperature", $\mathrm{H}$ ); taste ("some old time beekeepers... can taste that droplet of nectar and identify the flower that way, based on the taste", B1); hearing ("red belly woodpecker calling that's when a lot of my spring stuff is going [to be planted]... he's not gonna start mating calls till the weather is-till the light is in a certain way", G); and touch ("soft scale [pest]... exude a sugary substance called honeydew, so it's kind of a way of finding themwhen a lot of leaves are getting sticky", I). These instances illustrate how the human senses alone serve to inform participants of a range of processes and factors in the environment.

\section{Types of everyday biomarkers}

All 10 participants shared numerous experiences and habits of inferring environmental factors by observing elements of living systems. The everyday biomarkers used by participants can be classified as showing one specific factor (one-to-one); several possible factors (one-to-many); or the status of the ecosystem as a whole (ecosystem).

\section{One to one}

Biomarkers in this category map to concrete phenomena: a "chicken coop smell" in a beehive is used to detect foulbrood - a deadly bacterial infestation (B2); green water in a fish tank suggests an ozone deficiency ("I can look at this water and tell that the ozone system hasn't been working for 2 weeks on this... because it's green", A); particles resembling 'saw dust' at the bottom of vine plants help identify a squash vine borer pest (F1); hydrangea color (pink or blue) is matched with low or high soil $\mathrm{pH}(\mathrm{I})$; a piping sound signals "that a [new] queen is getting ready to emerge" and a colony will soon split (B1); accentuated leaf growth in fruit plants is correlated with "too much nitrogen in the soil" (F2). Input from these and a multitude of other one-to-one biomarkers is nearly always mapped to a single cause and a subsequent associated practice.

\section{One-to-many}

One-to-many biomarkers inform our participants of several possible factors as opposed to one conclusive state. For instance, interveinal chlorosis, a yellowing "between veins of leaves" suggests nutrient deficiency or $\mathrm{pH}$ imbalance (" $a$ lot of times pH effects the availability of nutrients", I); blossom end rot in tomatoes is "caused by a lack of calcium" (F1), or "the calcium's there but the plant isn't able to accept it because of the moisture content" (G); a 'sliming' fish suggests poor water quality or a parasite:

If a fish is sliming really heavily that's a good sign that something's wrong. It could be-water quality and the slime is trying to protect the animal [or] if there's a parasite that's causing the fish to slime really heavily to push that parasite off. (A)

Similarly, bees returning to the hive trembling indicate a contamination, but do not imply one specific chemical: 
If you have bees that are flying normally and coming back and trembling and dying they've obviously either got sprayed by something or got contaminated... (B2)

Thus, one-to-many biomarkers typically lead to further reflection and investigation on behalf of our participants in order to infer the cause or actions that should follow.

\section{Ecosystem}

Lastly, participants rely on numerous biomarkers to learn about the ecosystem as a whole. For example, B1 infers local drought and blooming cycles by observing his bees:

One of the neat things about beekeeping is that it kinda gets you in touch with your local ecosystem. When there's a drought I can tell, the bees aren't bringing in much nectar, you can tell when the bees are bringing in pollen by observing the hive so you know when the first flowers are blooming in the spring, you can tell what flowers they're working based on the color of the pollen. (B1)

Similarly, our IPM participant monitors the greenhouse by tracking the balance between pest and beneficial insects:

You always want to reach a balance, you never want to totally eradicate an insect... If you wanna sustain a population of beneficial insects, you always wanna have a baseline or a lower level of the pest insect - because they'll keep your beneficial [insect] around. (I)

Alternatively, organisms are also used to infer problems in the ecosystem: coral reef bleaching as "a response to stress, it's not necessarily any specific stress", (A); algae on reefs suggesting "a disturbance to the system... nutrients are very tightly cycled, algae indicates that they're not so tightly cycled", (A); the endangerment of the Philippine crocodile, suggesting "pollution, habitat loss, people kill them out of fear", (R); or diseases prevailing on unhealthy plants ("when a plant is stressed, a whole host of things can then be multiplied, diseases.. and pests will spread more easily", F2). These examples illustrate how biomarkers are used to learn about complex processes within the ecosystem or infer information about the ecosystem as a whole.

\section{Data collection, sharing and speculation}

Participants maintain a variety of records of their practice (Figure 4) including: daily logs of water quality and feedings (A, R); an extensive log and computer database of pest infestations (I); recipes of honey products (B1, B2); schedules and layouts for crop rotations (F1, F2, F3); or a gardening journal that combines planting information with mementos from personal life ("I'm hoping that my grandkids and great grandkids will be able to read that
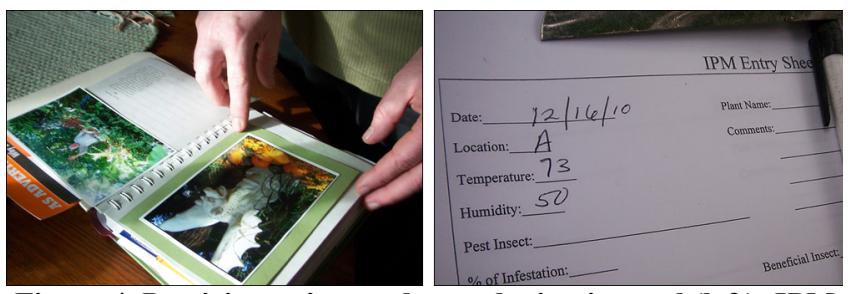

Figure 4. Participants' records: gardening journal (left); IPM data entry sheet (right). stuff", G). While G was hesitant to share his journal due to an interweaving of sentimental material with garden data, all other participants were eager to show us their records. However, in practice, they rarely saw value in sharing everyday data with people outside their work ("the only time that we really drew these graphs and all is when we were doing a PowerPoint doing a talk about the program", I; "what I do every day I don't think should be on the report- cleaning and feeding and the sort of mundane things", R). Records thus serve to inform aspects of the work (e.g., where to plant crops, what chemicals to add to the water), but are not typically shown to others.

\section{Sharing Mechanisms}

Nevertheless, participants do routinely share aspects of their work. Day to day information is discussed with other community members by word of mouth ("people call me... I lend out an extractor to people and they come and pick it up and they tell me what's going on", B1; "casual conversation", F1). More serious concerns, e.g. a mosquito spraying, might be posted to community listserves:

The mosquito spraying for instance, we put out a big email blast that said hey this is happening we're gonna monitor our hives, we suggest you do the same and we will let you know if anything came up we just kept everybody updated, we said nothings happening, nothings happening and everybody else reported the same... and it's good, watch out for each other. (B2)

Twitter and Facebook are utilized to broadcast community events ("tweet about things like- we're delivering this and this to the restaurant", F2); while mediums such as books, blogs and radio shows offer gardening information and advice to the general public $(\mathrm{G}, \mathrm{H})$. Alternatively, R speaks directly to zoo visitors to correct misunderstandings:

I'm always listening in on people when they talk and I try to interject with the correct information in a way that I don't insult them... maybe tell them more about the animal they didn't know and try to make them understand. (R)

Participants thus rely on a range of mediums, from conversation to Twitter and mailing lists, to share aspects of their practice in and outside of their communities.

\section{Speculation}

Participants tend to actively discuss the implications and causes of unusual environmental phenomena. For instance, all farming participants commented on a recent stinkbug infestation that affected the east coast of the United States. F2 wondered if it was caused by weather patterns and discussed the matter with other farmers:

I think there's something in the weather pattern that allowed them [stink bugs] to reproduce at those rates... a lot of farmers we spoke to had whole crops that were destroyed and that was very strange because we've never even seem the attack food crops. (F2)

Consequently, numerous community members began to experiment and share possible solutions to the problem: 
I got them on my blog-this woman sent me a picture [of] a mouse going onto her deck and eating stink bugs... then another woman called me... she gathered all the stinkbugs that she found and threw them out on the deck and here come the turkeys eating the stinkbugs. This is really great news, because to this point we didn't know if local birds would eat stinkbugs and now they're discovering it. (G)

Likewise, urban water quality emerged as an issue of concern for many participants: $G$ prefers to water his plants with rainwater ("just because there's no chlorine in it, fluoride and all that other crap", G), H uses a private well, and city water is filtered prior to entering A's aquariums. In particular, A notes the lack of accessible information about urban water ("you can call them and ask, or you can just test $\left.i t^{\prime}, \mathrm{A}\right)$, noting that certain chemicals are lethal for fish:

They [the city] can change the chemicals they use for disinfection [of water]... now a lot of facilities are switching to chloramine... so if it gets into your fish tank it really wreaks havoc. (A)

Moreover, the rise in colony collapse disorder - a national die-off in honeybees - has spurred a debate on pesticide use in beekeeping communities, locally and internationally. B2 showed us numerous posts on an online international forum (e.g., "document shows EPA allow bee toxic pesticide despite their own scientists' red flags", B2), suggesting pesticides and genetically modified plants as likely causes:

....all these self pollinating plants, or synthetically modified plants they don't need pollinators anymore, so that's taking away a food source, on top of that they're spraying the shit with chemicals that's killing the bees. (B2)

Both B1 and B2 also blame lack of government regulation ("In Europe they banned neonicotinoids because they believe it is hurting the bee population, but in this country, they haven't been able to prove it's a problem, but a lot of beekeepers think it is a problem", B1). Similarly, G speculates that commercial advertisements promoting stylized images of green lawns led people to use chemical sprays on their yards, resulting in nitrogen soil deficiencies ("clover takes the nitrogen out of the air and puts it in the soil... it wasn't until advertisers told us that we don't want clover in our lawns that were taught not to have clover in our lawns", G). To counter such advertisements, G endorses organic practices on his blog, radio show and in numerous publications: "this is what I teach people- there is no reason to use any chemical in your garden" (G).

Likewise, $\mathrm{R}$ is also taking action in his field - collaborating with another zoo to breed and reintroduce the endangered Louisiana Pine Snake into the wild. He notes the broader processes, which may have contributed to the endangerment:

They [Louisiana Pine Snakes] feed primarily on... the pocket gopher, and when it went they went with it, and the reason the pocket gopher went is cause they specialize in certain types of grass - a lightening would burn an area where these things would grow-the grass would grow first... well with forest management now there is no burning... that's what started the problems (R)
The above instances illustrate a range of reflections based on ambiguous or unexpected inputs from living systems. The resulting speculations are often projected to broader groups to infer potential meaning(s) as well as to consider (and in some cases even organize) collective action aimed at the responsible or intervening social and political forces.

\section{DISCUSSION}

Our findings detail the practices of 10 individuals who directly and indirectly work with living organisms on a daily basis. We highlight participants' proficiency with and access to a range of technologies, which range from social media such as mailing lists, blogs, forums and Twitter, to digital information systems such as a database of records or online weather reports, to highly technical sensors for soil and water quality. While our participants skillfully draw on these digital technologies throughout their practice, they also habitually rely on living systems (biomarkers) to infer information about the environment and shape their course of action. We highlight two unique themes that emerge from participants' use of everyday biomarkers.

\section{Biomarker systems}

Our findings suggest that biomarkers are perceived to be and used as integral components of larger systems. Some practices revolve around systems that are purely organic: designing a bioshelter "on the model of a living cell", or using one-to-many and ecosystem biomarkers (fish appearance, bee behavior, balance between pest and beneficial insects, etc.) to infer complex processes within an ecosystem, or gain a glimpse into its well-being as a whole. Other practices confirm or clarify 'naked' perceptions of living organisms with data from analog and digital tools, resulting in systems of interdependencies between technological and biological inputs. More broadly, participants' collective practices reveal larger, sociopolitical systems that shape their work: regional water quality treatment, national policies to preserve or destroy snake habitats, or international regulations on pesticide use.

\section{Active engagement with context}

Biomarkers not only cue participants to environmental states, but also serve as points of engagement with underlying contexts. In the most direct sense, participants physically interact with the environment. When tapping on plant leaves, tipping a beehive, inserting a finger into the soil, or diving into an aquarium, participants are active and immersed in their surroundings. On a higher level, participants become involved in the social and political processes that shape their practices. Examples such as beekeepers debating EPA regulations on international forums, farmers experimenting with natural remedies for stinkbugs, a gardener advising the general public against using commercially advertised chemical sprays, and a reptile keeper collaborating across state lines to preserve endangered snakes, all suggest that biomarkers serve to inspire active participation in broader contexts.

\section{DESIGN IMPLICATIONS AND OPPORTUNITIES}

We conclude with three design implications emerging from our data. We draw on our findings to expand the UbiComp 
community's conceptualization of 'sensor' and present new opportunity areas for the design of future sensing systems.

\section{Leveraging non-digital inputs}

Previously we outlined several popular categories of sensing in UbiComp and HCI research, whereby a sensor is typically conceptualized and studied as an electronic device. Our work suggests examples of both traditional tools (hand lens, etc.) and living organisms that may be construed as 'sensors'. Our participants effectively monitor factors such as soil temperature ("by seeing what's germinating"), ammonia levels (by smelling the water), amount of stored honey (by tipping the hive), or pest infestation levels (through counting and magnification tools) without the use of soil sensors, water quality monitors or IR insect tracking. When participants do rely on digital sensing, they often employ such technologies only as a secondary measure (e.g., chemical analysis of water when fish do not appear healthy; testing the soil if plants are not growing properly).

Our findings include a range of sensing practices that do not involve digital or electronic devices. As new research emerges to question technology-focused approaches in HCI (e.g., "could technology be replaced by an equally viable low-tech or non-technological approach?" [2]), and their implications (e.g., sustainable disposal of digital artifacts [5]), we argue for expanding the UbiComp community's vision of sensing beyond electronic devices, to include living organisms and traditional tools.

Throughout our fieldwork we discovered that sensors (digital, analog or organic) are rarely if ever used in isolation. Instead participants fluidly navigate across a hybrid system of biomarkers, traditional tools and digital devices to gain insights into environmental processes and inform future actions. Our findings suggest an opportunity to shift from designing sensing technologies to designing ubiquitous systems that incorporate living organisms and traditional tools along with digital devices. In particular, this paradigm could open a rich design space for active participatory sensing or passive environmental monitoring, for instance by expanding prior work in public air quality sensing (e.g., [1]) to integrate inputs from plants [24] and insects, or monitoring water quality (e.g., [22]) based on fish activity. Alternatively, entirely new ubiquitous systems could emerge to broaden our understanding of the ecosystem as a whole (rather than specific aspects such as water or air quality) by integrating inputs from plants, insects and animals along with analog tools and digital sensors into holistic representations of the environment. For instance, a system might track the well-being of a neighborhood by visualizing noise, air and water quality along with insect activity and appearance of street plants.

\section{Designing technologies that teach new ways of seeing}

Our data suggests that over time, participants developed a "zen feel" for the complex processes in their environment. Occasionally, highly skilled and nuanced ways of "reading" biomarkers were accompanied by the use of more advanced technologies. However, such technologies were sometimes abandoned after participants developed a skill (e.g., "working off how everything looks" instead of testing the soil), or were used infrequently and only to help resolve a problem that more traditional tools could not (e.g., D.O. meter to verify water quality when fish appear sick). These findings suggest a new model for designing digital sensors: sensing technologies as tools that support new ways of 'seeing' or engaging with the environment. Such sensors move away from 'smart' technology and towards systems that encourage human awareness, refection and wonderment about our living world [32].

Embracing low-fidelity signals. Digital sensing tools can offer remarkable degrees of precision, and the collected data lends itself to powerful methods of analysis. Graphs, charts and other scientific presentations are common throughout HCI sensing applications ranging from activity recognition to input sensing. These approaches contrast with the numerous 'imprecise' sensing practices of our participants: biomarkers (plant growth, reptile activity levels, etc.) and more traditional tools (e.g., monitoring tray) often provided highly useful and reliable information without the degrees of precision characteristic of digital sensors. Imprecision often prompted our participants' physical involvement with the materials being sensed: tipping a beehive, inserting a finger into soil, or tapping a plant leaf. These and similar actions can inspire future input techniques to support indiscreet and fluid interactions with digital systems. More broadly, ubiquitous systems can draw on 'imprecise' digital sensing to embrace "ambiguity as a resource for design" [17] or "design for doubt" [31]. For instance, as an alternative to providing concrete measurements such as time and duration of electrical appliance use [18], activity recognition systems might explore imprecise sensing to facilitate more critical explorations of human behavior. Similarly, research supporting outdoor learning experiences [e.g. 35] might use imprecise sensing to encourage inquiry into ambiguously represented environmental phenomena.

Peripheral engagement. The use of imprecise biomarkers and tools also inspired participants' engagement with context that is peripheral to the phenomena being sensed: by looking at larval stages, the IPM participant assessed the "balance" and well-being of the entire greenhouse; by tracking bee behavior, a beekeeper became "in touch with [the] local ecosystem"; by observing algae on a reef, A inferred a "disturbance to the system". While often subtle to articulate, this type of engagement seemed to play essential roles in developing a sensibility for understanding the environment. In addition, peripheral engagement was at times suggested as a source of meaning. Whether by directly "seeing something go from just a little ity bity thing all the way to fruition" or taking pride in the fact that "every third bite of food is attributed to the honey bee", participants drew experiential value from their practice.

These findings highlight the importance of the degree to which a digital sensor either facilitates or hinders peripheral engagement. For example, as noted in a recent CHI panel 
on food and sustainability [20], a system that senses soil moisture and waters plants remotely may discourage active presence in the garden. Consequently, the user may neglect or never learn important cues about the health of plants.

However, digital sensing also has the potential to support new and exciting forms of peripheral engagement, especially with phenomena that are difficult or impossible to sense with 'naked' human perception. For example, water quality sensing systems used in infrastructure and environmental sensing [e.g., 16] can reveal relationships between the home water system and local water source. Similarly, air quality monitoring can connect users with processes that contribute to air pollution in the home or neighborhood. On a higher level, considering peripheral engagement in the design of sensing systems can support more holistic interactions, including engagement with broader phenomena aside from ones directly being sensed.

Scaffolding. Through "years of experience" and insights from mentors, our participants developed highly nuanced sensibilities to infer information such as complex air or water quality from cues as subtle as a sliming fish or a trembling bee. With some participants viewing their practice as "an art or a craft", our findings suggest opportunities for new scaffolding tools that train individuals and groups to 'sense' better and differently. For instance, a body of research in activity recognition and participatory sensing appropriates mobile phones as digital sensing devices $[10$, etc. $]$. Complimentary to this work, mobile phones and other ubiquitous platforms can be used to direct people to their local environments, providing information that supports 'reading' natural or artificial biomarkers, such as appearances of plants and behaviors of animals. Some information technologies can serve as scaffolding tools that are needed less frequently or not at all after a sufficient level of skill has been developed. Others can be designed to nurture mentor/apprentice relationships in communities, encouraging sensing as a "conjoint practice" [26] and tool for community togetherness [14].

\section{Enriching practices of data collection and sharing}

Our participants routinely record environmental data in logs, databases, and personal journals. While these documents are not directly shown to other stakeholders, the participants actively share aspects of their practice, from day-to-day events discussed with local practitioners through "causal conversation", or telling zoo visitors "more about the animal", to broadcasting issues of local concern on community listserves and speculating about large-scale phenomena on international forums. As new citizen science and environmental monitoring applications continue to explore data sharing [e.g., 23, 21], we see a range of opportunities for collecting and presenting inputs from living systems and analog tools as well as digital sensors.

On one hand, we see opportunities for existing and future citizen science applications to incorporate a new user group: individuals working with living systems. Technologies can combine routine information collected by beekeepers, aquarists, reptile keepers, or gardeners with rich forms of metadata, for example: augmenting mite counts with observations of bee behavior; supplementing water quality data with images of fish appearance; integrating gardening data with mementos from personal life; or more broadly correlating inputs from organic systems with users' insights into the surrounding environmental processes. Alternatively, metadata might be embedded into the organic materials themselves (similar to augmenting fabric with storytelling [36]). For instance, a beehive could be annotated with current flower conditions or weather patterns; a plant bed might show crop rotation history, etc.

On a higher level, data collected from living organisms, coupled with personal or group annotations, can serve to further issues of community concern and political interest. As HCI research emerges to encourage collective action around shared issues [14], we see opportunities for supporting activism around the well-being of living systems (similar to technologies that sustain 'publics' [12]). For example, local beekeepers could capture and share videos of bee flights, attributing metadata to draw attention to potential pesticide spraying in the area (as per [7]). Similarly, gardeners and aquarists might upload images of fish and plants to track possible changes in urban water quality. Moreover, using the scaffolding tools outlined earlier, everyday citizens could be involved in the collection and sharing of biomarker data. Examples include mobile applications that enable photographs and annotations of nature reserves to assess snake habitats, or insect counting tools to track local pest populations. Resulting data could be shared within and across communities of local residents, individuals working with living systems, or government officials thus supporting "politics of scale" [15] by linking people through their collective actions in the service of broader social change.

\section{CONCLUSION}

We presented the environmental monitoring practices of 10 individuals who routinely engage with living organisms. Our participants draw on independent and interrelated systems of biomarkers, traditional tools and digital sensors to infer information about the environment and inform actions related to local ecosystems. We use our findings to reflect on current sensing paradigms and explore approaches for incorporating living organisms, traditional tools and digital devices into future sensing systems. We propose (1) leveraging non-digital inputs in ubiquitous systems, (2) designing technologies that teach new ways of 'seeing', and (3) enriching practices of data collection and sharing as opportunities for expanding and guiding future research in this area. Ultimately, we hope our work inspires a broader framing of and a more hybrid approach towards sensing infrastructures that support the diversity and richness of human and environmental ecosystems.

\section{ACKNOWLEDGEMENTS}

Financial support for this research was provided in part by the Vira I. Heinz Endowment and the National Science Foundation under Grant No. 1018340. We are deeply thankful to Alex Taylor and our reviewers for their 
thoughtful and constructive feedback, which will continue to shape our future research.

\section{REFERENCES}

1. Aoki, P. et al. 2009. A Vehicle for Research: Using Street Sweepers to Explore the Landscape of Environmental Community Action. Proc. of CHI '09.

2. Baumer E., Silberman, S. 2011. When the implication is not to design (technology). Proc. of CHI 2011.

3. Beckwith, R., Teibel, D., Bowen, P. 2004. Report from the Field: Results from an Agricultural Wireless Sensor Network. In Proceedings of LCN '04, 471-478.

4. Bidwell, N., Browning, D. 2010. Pursuing genius loci: interaction design and natural places. Personal Ubiquitous Comput. 14, 1 (January 2010), 15-30.

5. Blevis, E. 2007. Sustainable interaction design. CHI '07

6. Burrell, J., Brooke, T., Beckwith, R. 2004. Vineyard Computing: Sensor Networks in Agricultural Production. IEEE Pervasive Computing 3, 1 (January 2004), 38-45.

7. J. Campbell, L. Mummert, R. Sukthankar. Video Monitoring of Honey Bee Colonies at the Hive Entrance. Visual observation \& analysis of animal \& insect behavior, ICPR'08.

8. Card, S. K., Mackinlay, J. D., Robertson, G. G. 1990. The design space of input devices. CHI ' 90.

9. Chetty, M., Tran, D., Grinter, R. E. 2008. Getting to green: understanding resource consumption in the home. UbiComp'08.

10. Consolvo, S., et al. 2008. Activity sensing in the wild: a field trial of ubifit garden. CHI '08.

11. Dillahunt, T., Mankoff, J., Paulos, E., Fussell, S.. 2009. It's not all about "Green": energy use in low-income communities. In Proceedings of UbiComp '09.

12. DiSalvo, C. 2009. Design and the Construction of Publics. Design Issues, Vol. 25, No. 1 (2009): 48-63.

13. DiSalvo, C., Boehner, K., Knouf, N. A., Sengers, P. Nourishing the Ground for Sustainable HCI: Considerations from Ecologically Engaged Art. CHI '09.

14. DiSalvo, C. Marti, L., Coupland, J., Steiner, M. A 2009. Local Issues, Local Uses: Tools for robotics and sensing in community contexts. C\&C'09: Creativity \& Cognition.

15. Dourish, P. 2010. HCI and Environmental Sustainability: The Politics of Design and the Design of Politics. DIS'10

16. Froehlich, J. E., et al. 2009. HydroSense: infrastructuremediated single-point sensing of whole-home water activity. In Proceedings of UbiComp '09.

17. Gaver, W. W., Beaver, J., Benford, S. 2003. Ambiguity as a resource for design. In Proc. of the SIGCHI '03.

18. Gupta, S., Reynolds, M. S., Patel, S. N. 2010. ElectriSense: single-point sensing using EMI for electrical event detection and classification in the home. UbiComp '10.

19. Harrison, C. Desney Tan, and Dan Morris. 2010. Skinput: appropriating the body as an input surface. CHI '10.

20. Hirsch, T., Sengers, P., Blevis, E., Beckwith, R., Parikh, T. 2010. Making food, producing sustainability. CHI EA '10.

21. Jeremijenko, N., Arnold, J. J., Kavesh, W. Feral Robotic Dogs. 2009.
22. Kim, S., Robson, C., Zimmerman, T., Harber, E., Pierce, J. 2011. Creek Watch: Pairing Usefulness and Usability for Successful Citizen Science. CHI 2011

23. Kim, S., Paulos, E. inAir: Sharing Indoor Air Quality Measurements and Visualizations. CHI 2010

24. Kuribayashi, S., Sakamoto, Y., Tanaka, H. 2007. I/O plant: a tool kit for designing augmented human-plant interactions. Ext. Abs. of of CHI '07, 2537-2542.

25. Kurtenbach, G., Buxton, W. 1991. Issues in combining marking and direct manipulation techniques. In Proceedings of UIST ' 91.

26. Kuznetsov, S., Davis, G. N., Cheung, J. C., Paulos, E. 2011. Ceci N'est Pas Une Pipe Bombe: Authoring Urban Landscapes with Air Quality Sensors. CHI 2011, to appear

27. Lee, M.L., Dey, A.K. 2008. Lifelogging Memory Appliance for People with Episodic Memory Impairment. Proceedings of UbiComp'08.

28. Leshed, G., Velden, T., Rieger, O., Kot, B., Sengers, P. 2008. In-car gps navigation: engagement and disengagement from the environment. CHI '10, 1675-1684.

29. Odom, W. 2010. "Mate, we don't need a chip to tell us the soil's dry": opportunities for designing interactive systems to support urban food production. In Proc. of DIS ' 10.

30. Paxton, M., Benford, S. 2009. Experiences of participatory sensing in the wild. Proc of UbiComp '09, 265-274.

31. Paulos, E. 2009. Designing for Doubt: Citizen Science and the Challenge of Change. Engaging Data, MIT, Cambridge.

32. Paulos, E., Jenkins, T., Joki, A., Vora. P. 2008. Objects of wonderment. DIS '08, New York, NY, USA, 350-359.

33. Pearce, J., Smith, W., Nansen, B., Murphy, J. 2009. Smart Garden Watering: experiences of using a garden watering simulation. Proc. of OZCHI '09, 217-224.

34. Rana, R. K., Chou, C. T., Kanhere, S. S., Bulusu, N., Hu, W. (2010). Ear-phone: an end-to-end participatory urban noise mapping system. ACM/IEEE IPSN '10.

35. Rogers, Y. et al. 2004. Ambient wood: designing new forms of digital augmentation for learning outdoors. IDC '04, 3-10.

36. Rosner, D. K., Ryokai, K. 2008. Spyn: augmenting knitting to support storytelling and reflection. UbiComp '08.

37. Ruiz-Altisent, M. et al. 2010. Review: Sensors for product characterization and quality of specialty crops-A review. Comput. Electron. Agric. 74, 2 (November 2010), 176-194.

38. Ryokai et al. GreenHat: Exploring the Natural Environment Through Experts' Perspectives. CHI '11.

39. Shklovski, I., Vertesi, J., Troshynski, E., Dourish, P. 2009. The commodification of location: dynamics of power in location-based systems. In Proceedings of UbiComp '09.

40. Willett, W., Aoki, P., Kumar, N., Subramanian, S., Woodruff, A. (2010) Common Sense Community: Scaffolding Mobile Sensing and Analysis for Novice Users. Pervasive'10

41. Woodruff, A., Hasbrouck, J., Augustin, A. 2008. A bright green perspective on sustainable choices. SIGCHI'08.

42. Wyche, S. P., Magnus, C. M., Grinter, R. E. 2009. Broadening UbiComp's vision: an exploratory study of charismatic pentecostals and technology use in Brazil. In UbiComp '09 